\title{
Effects of short-term atorvastatin use in patients with calcium stones: A randomized placebo- controlled clinical trial
}

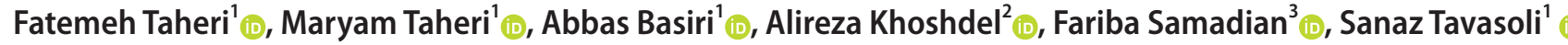 \\ 'Urology and Nephrology Research Center, Shahid Beheshti University of Medical Sciences, Tehran, ${ }^{2}$ Modern Epidemiology Research Centre, Aja University of Medical \\ Science, Tehran, ${ }^{3}$ Urology and Nephrology Research Center, Shahid Labbafinejad Medical Center, Shahid Beheshti University of Medical Sciences, Tehran, Iran
}

Purpose: A few experimental and observational studies have reported that atorvastatin prevents calcium oxalate stone formation. Our study is the first to investigate the effect of atorvastatin on 24-hour urinary metabolites, urinary malondialdehyde (U-MDA) (an oxidative stress marker) and urinary neutrophil gelatinase-associated lipocalin (U-NGAL) (a renal tubular injury marker) in patients with calcium stones and hyperoxaluria.

Materials and Methods: This randomized, double-blind, placebo-controlled, parallel-group clinical trial included 32 adults with recurrent calcium stone formation and hyperoxaluria. All participants received a 3-month course of either atorvastatin $(20 \mathrm{mg} /$ d) or placebo of an identical shape. Both groups received the usual nutritional care based on the European Association of Urology guidelines.

Results: Twenty-eight participants completed the study. Serum levels of total and low-density lipoprotein cholesterol decreased in the atorvastatin group, and these changes were significantly different between groups $(p<0.001)$. No statistically significant differences were observed between intergroup changes of the 24-hour urinary metabolite analysis, the U-MDA to creatinine ratio and the U-NGAL to creatinine ratio.

Conclusions: Atorvastatin administration at a dose of $20 \mathrm{mg} / \mathrm{d}$ for 3 months did not affect 24-hour urinary metabolite, U-MDA and U-NGAL levels in recurrent calcium stone formers. However, this study could not disprove the preventive role of atorvastatin in kidney stone formation. Future studies should consider a larger sample size, longer follow-up, different drug doses, and measurements of multiple biomarkers of oxidative stress and tubular injury.

Keywords: Hydroxymethylglutaryl-CoA reductase inhibitors; Lipocalin-2; Malondialdehyde; Oxidative stress; Urolithiasis

This is an Open Access article distributed under the terms of the Creative Commons Attribution Non-Commercial License (http://creativecommons.org/licenses/by-nc/4.0) which permits unrestricted non-commercial use, distribution, and reproduction in any medium, provided the original work is properly cited.

\section{INTRODUCTION}

Calcium oxalate $(\mathrm{CaOx})$ stones are the most prevalent type of kidney stones worldwide and also in Iran [1]. The pathogenesis of $\mathrm{CaOx}$ stones is multifactorial, and this con- dition is associated with several chronic diseases, such as cardiovascular disease, diabetes mellitus, and dyslipidemia. Reportedly, dyslipidemia increases the risk of kidney stone formation [2]. A few studies have reported that statin intake could protect against kidney stone formation [3,4].

Received: 26 May, 2019 - Accepted: 17 July, 2019

Corresponding Author: Sanaz Tavasoli (iD) https://orcid.org/0000-0001-7561-3918

Urology and Nephrology Research Center, Shahid Beheshti University of Medical Sciences, No.103, Shahid Jafari St., Pasdaran Ave., Tehran, Iran TEL: +98-2122567222, FAX: +98-2122567282, E-mail: s.tavasoli@sbmu.ac.ir 
Statins are a group of lipid-lowering drugs, which act via inhibition of 3-hydroxy-3-methylglutaryl coenzyme A reductase. In addition to their lipid-lowering action, they are known to decrease the risk of chronic diseases, such as cardiovascular disease and different types of cancers [4]. Sur et al. [3] and Cohen et al. [4] investigated the effect of statins on urolithiasis in humans, and both studies reported that statin use was associated with a reduced risk of stone formation. However, the underlying mechanisms contributing to this effect remain unclear. The study by Cohen et al. [4] suggested that the effect is independent of the lipid-lowering action of statins. Two experimental studies by Tsujuhata et al. $[5,6]$ performed in rat models of hyperoxaluria and $\mathrm{CaOx}$ stones reported that atorvastatin administration inhibited renal crystal retention by decreasing oxidative stress caused by oxalate and $\mathrm{CaOx}$ crystals. A recent experimental study performed by Temiz et al. [7] in a rat model of urolithiasis reported that atorvastatin administration significantly decreased uric acid but increased calcium levels. To our knowledge, no clinical trial has investigated the effect of atorvastatin in patients with kidney stones. This current study investigated the effects of short-term atorvastatin use in patients with calcium stones and hyperoxaluria. We investigated the effects of the drug on 24-hour (h) urinary metabolites and also urinary malondialdehyde (U-MDA) (a marker of oxidative stress) and urinary neutrophil gelatinase-associated lipocalin (U-NGAL) (a marker of renal tubular injury).

\section{MATERIALS AND METHODS}

\section{Trial design and participants}

This study was a randomized, double-blind, placebocontrolled, parallel-group clinical trial with an allocation ratio of 1:1. All participants were recruited from the Stone Prevention Clinic at Shahid Labbafinejad Hospital, Tehran, Iran. Recurrent calcium stone formers (those with a history of at least 2 episodes of radiopaque stone formation [8] with hyperoxaluria [ $24 \mathrm{~h}$ urinary oxalate $>40 \mathrm{mg} / 24 \mathrm{~h}$ ]) were enrolled. Other inclusion criteria included the following: age 18 to 65 years, competency to provide informed consent, and no surgical interventions performed within 20 days preceding study enrollment [8]. Exclusion criteria were as follows: primary or enteric hyperoxaluria (urinary oxalate $>80 \mathrm{mg} / 24$ $\mathrm{h}$ ), new prescriptions or changes in the dose of thiazides, use of medications that could affect urinary oxalate homeostasis including calcium, magnesium, and potassium supplements and pyridoxine, use of antioxidant or anti-inflammatory drugs or supplements (e.g, steroids, nonsteroidal anti-inflammatory drugs, pioglitazone, and/or multivitamins containing vitamin $\mathrm{A}, \mathrm{C}$, or E), a history of diabetes mellitus, hepatic failure, thyroid or parathyroid diseases, chronic kidney disease, urinary tract infection, chronic diarrhea, cancers, or immunological diseases, and pregnancy or lactation.

\section{Sample size, randomization, and blinding}

As mentioned earlier, no previous research was available as a reference for sample size estimation for this study. Differences in U-MDA levels were used as the primary endpoint to calculate the sample size. Considering an effect size of 1 (based on a study reported by Chow et al. [9] and the limitations regarding the feasibility of the project at our center), as well as a type I error ( $\alpha$ ) of 0.05 and type $\Pi$ error ( $\beta$ ) of 0.2 , the required number of participants was determined as 16 per group. Permuted-block randomization was used to categorize participants into blocks with a block size of 4 . A computer program was used to generate a random sequence before patient allocation. Both, participants and investigators were blinded to the study allocation. The drug and placebo containers were identical in shape and were sequentially coded by one of the study investigators. The codes were kept secret until data analysis was completed.

All participants provided written informed consent, and the study was performed in accordance with the 1964 Declaration of Helsinki. The Ethics Committee of the Urology and Nephrology Research Center at Shahid Beheshti University of Medical Sciences approved the study (reference number: 931223-10). The trial was registered in the Iranian Registry of Clinical Trials (IRCT) (IRCT registration number: IRCT2017021826406N2).

\section{Interventions}

All participants received a 3-month course of either atorvastatin $(20 \mathrm{mg} / \mathrm{d})$ or placebo. Both groups received the usual nutritional care at the Stone Prevention Clinic based on the European Association of Urology guidelines, including normal calcium but restricted oxalate intake [10]. Participants were instructed to return forgotten pills at the end of the study period to evaluate drug compliance. The ratio of consumed to total pills was used as a measure of drug compliance [11].

\section{Outcomes and data collection techniques}

Study outcomes included levels of 24-h urinary metabolites, U-MDA and U-NGAL. As mentioned earlier, U-MDA was assessed as a marker of oxidative stress and U-NGAL as a marker of renal tubular injury. Other variables investigated were anthropometric indices, fasting serum glucose, and the lipid profile. All variables were investigated at the 
beginning and at the end of the study.

In this study, 24-h urinary metabolites were measured using the method described by previous reports [12] and included evaluation of creatinine, uric acid, calcium, magnesium, phosphate, sodium, potassium, citrate, and oxalate. The $\mathrm{CaOx}$ supersaturation, calcium phosphate supersaturation, and uric acid supersaturation values were calculated using the LithoRisk software (Biohealth, Italy) based on urinary metabolite measurements in 24 -h urine samples.

The first morning urine sample was collected for the measurement of U-MDA, U-NGAL, and creatinine. All urine samples were collected in sterile containers under aseptic precautions and centrifuged to remove any particulate matter. The samples were stored at $-80^{\circ} \mathrm{C}$ until they were analyzed. The Kei Satoh method was used to measure U-MDA [13], and measurements were performed within 6 months after sampling. The sandwich ELISA method was used for measurement of U-NGAL with the R\&D Systems DuoSet Kit DY1757 (Minneapolis, MN, USA). U-MDA and U-NGAL levels were divided by urinary creatinine levels to normalize for changes in urine concentration.

Blood samples were obtained following a 12-h overnight fast, and serum was separated for analysis. Participants' weight, height, and waist circumference were measured as previously reported [14], and the body mass index was expressed as $\mathrm{kg} / \mathrm{m}^{2}$.

\section{Statistical analysis}

All statistical analyses were performed using the IBM SPSS Statistics for Windows software ver. 22.0 (IBM Corp., Armonk, NY, USA). Normality of data was analyzed using the Shapiro-Wilk test. Data with skewed distribution were analyzed using nonparametric tests. Differences in baseline or final data between two groups were analyzed using the independent t-test (Mann-Whitney U test for data showing skewed distribution) for numerical continuous data and the chi-square test for categorical data. Intergroup differences in all dependent variables were calculated and compared between groups using the independent t-test (Mann-Whitney $U$ test for data showing skewed distribution). A p-value $<0.05$ was considered statistically significant.

\section{RESULTS}

This study included 32 participants and was performed between May 2016 and January 2017. Participants were randomly allocated to the aforementioned study groups, and eventually, 28 participants completed the study. All participants consumed $>80 \%$ of prescribed medication (mean, 90.1; 95\% confidence interval, 87.0-93.2). The CONSORT participant flow diagram is presented in Fig. 1.

Demographic and participants' baseline data, as well as serum parameters of participants are presented in Tables 1 and 2, respectively. Study groups were not different in
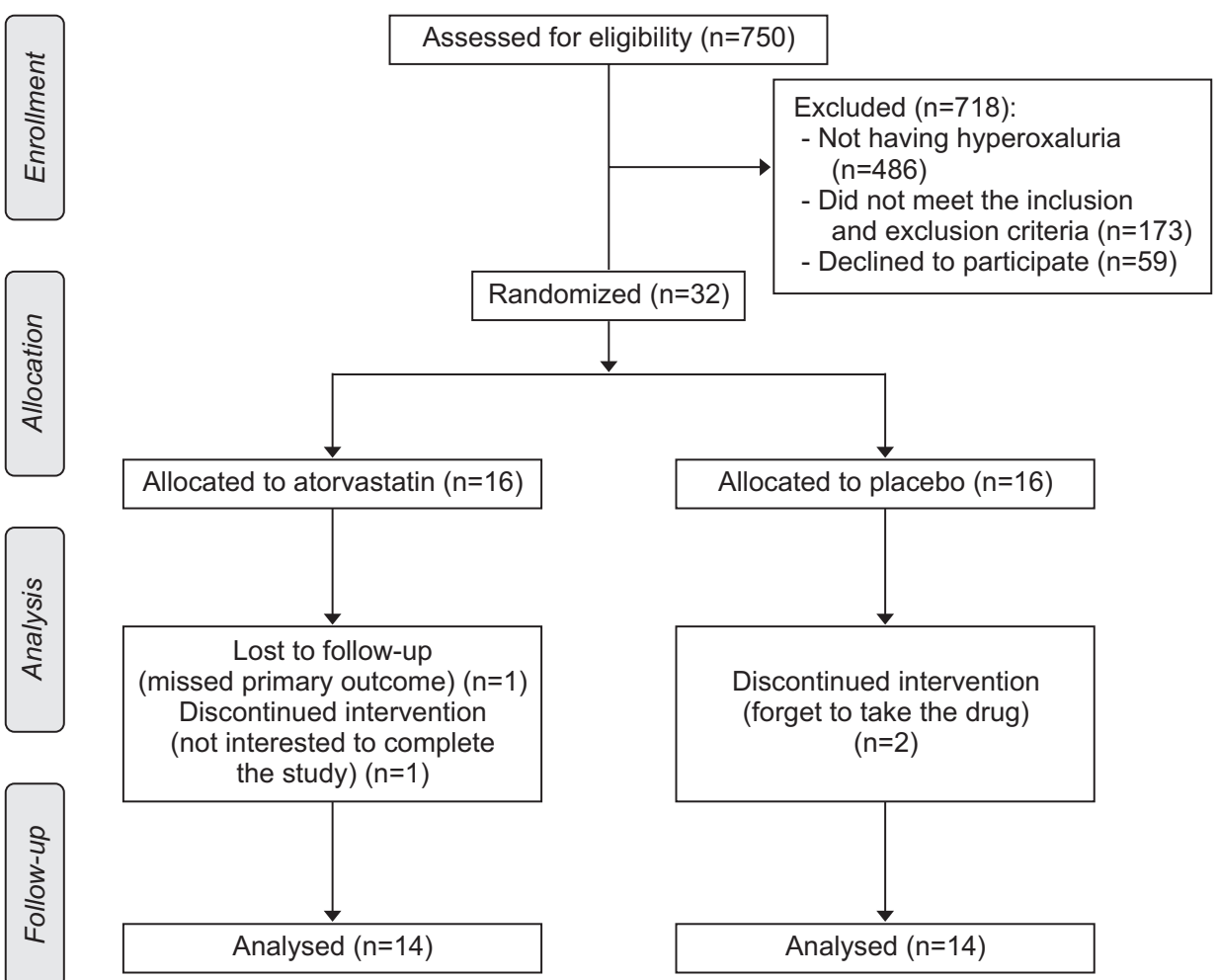

Fig. 1. Participant flow diagram. 
baseline patient characteristics except with regard to serum triglyceride (TG) levels $(\mathrm{p}=0.015)$.

We observed that the levels of serum total and lowdensity lipoprotein cholesterol decreased in the atorvastatin group, and these intergroup changes were significantly different between groups ( $p<0.001$, for both analyses). The level of serum TG levels was also decreased in atorvastatin group; however, the intergroup changes were nearly but not statistically different between groups $(p=0.057$ ). No significant differences were observed between study groups with regard to the intergroup changes in serum levels of other variables.

Results of 24-h urinary metabolite analysis are presented in Table 3. No significant differences were observed in intergroup changes with regard to 24 -h urinary metabolites.

The pre- and post-intervention results of the U-MDA to creatinine ratio (U-MDA/Cr) and the U-NGAL to creatinine ratio (U-NGAL/Cr) are presented in Table 4. No significant differences were observed with regard to intergroup changes in the U-MDA/Cr and U-NGAL/Cr values.

\section{DISCUSSION}

This study highlighted that atorvastatin administration (20 mg/d) for 3 months did not affect 24-h urinary metabolites, as well as U-MDA/Cr and U-NGAL/Cr values. To our knowledge, this randomized clinical trial is the first study to investigate the effect of atorvastatin on urinary metabolites or oxidative and tubular injury markers in patients with recurrent nephrolithiasis. Sur et al. [3] and Cohen et al. [4] investigated the association between statin use and urolithiasis; however, data regarding 24-h urinary metabolites were not available in their studies. Temiz et al. [7] reported that atorvastatin administration in a rat model of urolithiasis significantly decreased uric acid levels but increased calcium levels [7]. In contrast, Tsujihata et al. [5] reported that atorvastatin administration in a rat model of kidney stones and hyperoxaluria was not associated with changes in urinary oxalate or calcium levels. Santhosh Kumar et al. [15] investigated the effect of vitamin $\mathrm{E}$ and selenium administration (antioxidant and anti-inflammatory agents, respectively) in an animal model of hyperoxaluria and reported a significant reduction in urinary oxalate levels. They also observed a decrease in the specific activity of glycolic acid oxidase, lactate dehydrogenase, and xanthine oxidase in the kidney and liver tissue of the experimental animals. These enzymes participate in the synthesis of free radicals and oxalate [16]; therefore, antioxidant induced-suppression of these enzymes could have contributed to reduced oxalate synthesis. However, these results were not confirmed by our study. With regard to oxidative and tubular injury markers, our results do not concur with those of previous experimental studies [5,6], which reported that atorvastatin administration decreased urinary levels of N-acetyl- $\beta-\mathrm{D}$-glucosaminidase (NAG) (a marker of tubular injury) and 8-hydroxydeoxyguanosine (8-OHdG) (a biomarker of oxidative stress) in a rat model of hyperoxaluria and kidney stones. Our results are also in contrast to results of the retrospective epidemiological studies performed by Sur et al. [3] and Cohen et al. [4], which reported that long-term statin administration was associated with a reduced risk of urinary stones in patients with hyperlipidemia. Discrepancies between animal or epidemiological studies and clinical trials have been reported by previous studies investigating the effect of vitamin $\mathrm{E}$ or beta-carotene on cardiovascular diseases $[17,18]$. The contradictory results

Table 1. Demographic data and baseline patient characteristics

\begin{tabular}{lccc}
\hline \multicolumn{1}{c}{ Characteristic } & Placebo group $(\mathbf{n}=14)$ & Atorvastatin group $(\mathbf{n}=14)$ & $\mathbf{p}$-value \\
\hline Age $(\mathrm{y})$ & $46.6 \pm 11.1$ & $47.8 \pm 9.1$ & 0.768 \\
Sex & & & 0.663 \\
Female & $4(28.6)$ & $11(78.6)$ & $21.4)$ \\
Male & $10(71.4)$ & $28.90 \pm 4.77$ & 0.965 \\
Body mass index $\left(\mathrm{kg} / \mathrm{m}^{2}\right)$ & $28.82 \pm 3.69$ & $102.0 \pm 8.4$ & 0.523 \\
Waist circumference $(\mathrm{cm})$ & $99.5 \pm 11.8$ & $4(28.6)$ & 0.303 \\
Current smoking history & $2(14.3)$ & $5(35.7)$ & 0.403 \\
Metabolic syndrome & $3(21.4)$ & $116.8 \pm 124.2$ & $0.943^{\mathrm{a}}$ \\
Disease duration (mo) & $138.9 \pm 148.2$ & $5.8 \pm 9.5$ & $0.936^{\mathrm{a}}$ \\
Episodes of stone formation & $4.7 \pm 5.5$ & $122.18 \pm 13.79$ & 0.829 \\
Systolic blood pressure $(\mathrm{mm} \mathrm{Hg})$ & $123.35 \pm 13.04$ & $78.82 \pm 11.58$ & $0.166^{\mathrm{a}}$ \\
Diastolic blood pressure $(\mathrm{mm} \mathrm{Hg})$ & $71.07 \pm 18.52$ & & \\
\hline
\end{tabular}

Values are presented as mean \pm standard deviation or number (\%).

${ }^{\mathrm{a}}$ :Nonparametric analysis. 
Table 2. Serum parameters of study participants before and after intervention

\begin{tabular}{|c|c|c|c|}
\hline Variable & Placebo group $(n=14)$ & Atorvastatin group $(n=14)$ & p-value \\
\hline \multicolumn{4}{|l|}{ Total cholesterol (mg/dL) } \\
\hline Baseline & $183.1 \pm 28.8$ & $201.2 \pm 32.3$ & 0.16 \\
\hline End of trial & $186.9 \pm 26.0$ & $141.9 \pm 35.3$ & 0.001 \\
\hline Difference between baseline and end-of-trial levels & $3.8(-8.3$ to 15.9$)$ & $-59.3(-72.5$ to -46.0$)$ & $<0.001^{* * *}$ \\
\hline \multicolumn{4}{|l|}{ Low-density lipoprotein cholesterol (mg/dL) } \\
\hline Baseline & $105.5 \pm 17.4$ & $112.5 \pm 24.2$ & 0.47 \\
\hline End of trial & $105.7 \pm 17.7$ & $70.8 \pm 26.0$ & $<0.001^{* * *}$ \\
\hline Difference between baseline and end-of-trial levels & $0.2(-7.5$ to 7.8$)$ & $-41.7(-51.7$ to -31.7$)$ & $<0.001^{* * *}$ \\
\hline \multicolumn{4}{|l|}{ High-density lipoprotein cholesterol (mg/dL) } \\
\hline Baseline & $47.5 \pm 14.3$ & $46.5 \pm 9.1$ & 0.99 \\
\hline End of trial & $47.6 \pm 9.8$ & $45.9 \pm 7.4$ & 0.49 \\
\hline Difference between baseline and end-of-trial levels & $0.2(-4.4$ to 4.7$)$ & $0.6(-5.0$ to 3.8$)$ & 0.8 \\
\hline \multicolumn{4}{|l|}{ Triglycerides (mg/dL) } \\
\hline Baseline & $133.5 \pm 30.8$ & $196.6 \pm 63.3$ & $0.015^{*}$ \\
\hline End of trial & $130.6 \pm 38.5$ & $143.9 \pm 60.9$ & 0.31 \\
\hline Difference between baseline and end-of-trial levels & $-2.9(-22.2$ to 16.5$)$ & $-52.7(-105.7$ to 0.4$)$ & 0.057 \\
\hline \multicolumn{4}{|l|}{ Urea (mg/dL) } \\
\hline Baseline & $27.8 \pm 8.5$ & $31.4 \pm 6.2$ & 0.21 \\
\hline End of trial & $31.6 \pm 7.9$ & $30.6 \pm 6.7$ & 0.63 \\
\hline Difference between baseline and end-of-trial levels & $3.8(-2.1$ to 9.7$)$ & $-0.9(-4.0$ to 2.3$)$ & 0.13 \\
\hline \multicolumn{4}{|l|}{ Creatinine (mg/dL) } \\
\hline Baseline & $1.08 \pm 0.15$ & $1.15 \pm 0.17$ & 0.25 \\
\hline End of trial & $1.13 \pm 0.17$ & $1.17 \pm 0.15$ & 0.54 \\
\hline Difference between baseline and end-of-trial levels & $0.05(-0.01$ to 0.10$)$ & $0.01(-0.06$ to 0.09$)$ & 0.46 \\
\hline \multicolumn{4}{|l|}{ Fasting blood glucose(mg/dL) } \\
\hline Baseline & $98.0 \pm 8.3$ & $95.3 \pm 10.3$ & 0.62 \\
\hline End of trial & $100.4 \pm 13.2$ & $95.25 \pm 8.0$ & 0.39 \\
\hline Difference between baseline and end-of-trial levels & $2.4(-3.0$ to 7.8$)$ & $-0.1(-5.6$ to 5.4$)$ & 0.48 \\
\hline
\end{tabular}

Values are presented as mean \pm standard deviation or $95 \%$ confidence interval.

${ }^{*} \mathrm{p}<0.05,{ }^{* * *} \mathrm{p}<0.001$.

across studies could be attributed to the lower clinical dose of the medication administered, concomitant effects of other factors, short duration of intervention and follow-up in human studies, differences in patient compliance, and exclusion of high-risk patients [11,17,18]; however, the exact reason remains unclear. Notably, some studies have suggested that the lack of expected effects observed in clinical trials does not disprove the role of oxidative stress in several diseases such as cardiovascular diseases [17,18].

Selecting an appropriate dose and duration of treatment is challenging in clinical trials. We used a single dose of atorvastatin, which serves as a limitation of our study. Studies performed by Sur et al. [3] and Cohen et al. [4] used different drugs belonging to the statin group with different doses; however, the authors could not draw definitive conclusions regarding the optimal dose of these drugs. Therefore, an optimal type of statin and its dose remain undetermined. With regard to the appropriate duration of therapy, the median follow-up period in the study performed by Sur et al. [3] was 36.3 months in men and 47.2 months in women. The followup period in the study performed by Cohen et al. [4] was 4 to 6 years. Oxidative stress-induced formation of kidney stones is typically a gradual process that occurs over prolonged periods of time [19]. Therefore, an adequately long follow-up period is necessary in studies investigating kidney stone formation to confirm the effectiveness of any intervention.

An important concern in this study is the limitations of the biomarkers used to measure oxidative stress and tubular injury. Oxidative stress and tubular injury cannot directly be measured in the kidney tissue in most cases; therefore, human studies rely on the measurement of surrogate biomarkers in blood and urine in patients with kidney stones. U-MDA [20], 8-OHdG [21], and isoprostane [22] are among the surrogate biomarkers that measure oxidative stress, and urinary NGAL [23,24], gamma-glutamyl transpeptidase [25], and NAG [26] serve as markers of tubular injury. The discrep- 
Table 3. Results of measurement of 24-hour urinary metabolites before and after intervention

\begin{tabular}{|c|c|c|c|}
\hline Variable & Placebo group $(n=14)$ & Atorvastatin group $(n=14)$ & p-value \\
\hline \multicolumn{4}{|l|}{ Urinary volume $(\mathrm{mL} / 24 \mathrm{~h})$} \\
\hline Baseline & $2,279 \pm 740$ & $2,332 \pm 405$ & $0.23^{\mathrm{a}}$ \\
\hline End of trial & $2,486 \pm 1,197$ & $2,386 \pm 331$ & $0.14^{\mathrm{a}}$ \\
\hline Difference between baseline and end-of-trial levels & $207(-375$ to 789$)$ & $54(-146$ to 253$)$ & $0.98^{\mathrm{a}}$ \\
\hline \multicolumn{4}{|l|}{ Creatinine (g/24 h) } \\
\hline Baseline & $1.31 \pm 0.40$ & $1.31 \pm 0.59$ & 0.97 \\
\hline End of trial & $1.37 \pm 0.46$ & $1.26 \pm 0.38$ & 0.48 \\
\hline Difference between baseline and end-of-trial levels & $0.06(-0.07$ to 0.20$)$ & $-0.05(-0.29$ to 0.20$)$ & 0.41 \\
\hline \multicolumn{4}{|l|}{ Calcium (mg/24 h) } \\
\hline Baseline & $219.0 \pm 71.4$ & $259.9 \pm 79.6$ & 0.17 \\
\hline End of trial & $248.5 \pm 58.5$ & $235.5 \pm 132.3$ & $0.15^{\mathrm{a}}$ \\
\hline Difference between baseline and end-of-trial levels & $29.5(-25.5$ to 84.5$)$ & $-24.4(-115.6$ to 66.9$)$ & 0.29 \\
\hline \multicolumn{4}{|l|}{ Phosphorus (g/24 h) } \\
\hline Baseline & $0.76 \pm 0.25$ & $0.77 \pm 0.20$ & 0.89 \\
\hline End of trial & $0.76 \pm 0.24$ & $0.77 \pm 0.27$ & $0.72^{\mathrm{a}}$ \\
\hline Difference between baseline and end-of-trial levels & $0.01(-0.15$ to 0.16$)$ & $0(-0.15$ to 0.16$)$ & 0.97 \\
\hline \multicolumn{4}{|l|}{ Magnesium (mg/24 h) } \\
\hline Baseline & $99.0 \pm 45.2$ & $85.3 \pm 33.5$ & $0.77^{\mathrm{a}}$ \\
\hline End of trial & $109.6 \pm 41.0$ & $105.7 \pm 38.3$ & 0.79 \\
\hline Difference between baseline and end-of-trial levels & $10.6(-8.9$ to 30.1$)$ & 20.4 (4.6 to 36.2$)$ & $0.15^{\mathrm{a}}$ \\
\hline \multicolumn{4}{|l|}{ Sodium (mEq/24 h) } \\
\hline Baseline & $168.6 \pm 50.3$ & $191.5 \pm 70.1$ & 0.33 \\
\hline End of trial & $178.3 \pm 72.6$ & $175.5 \pm 64.7$ & 0.91 \\
\hline Difference between baseline and end-of-trial levels & $9.7(-34.5$ to 54.0$)$ & $-16.0(-53.6$ to 21.6$)$ & 0.35 \\
\hline \multicolumn{4}{|l|}{ Potassium (mEq/24 h) } \\
\hline Baseline & $70.3 \pm 30.6$ & $64.9 \pm 21.7$ & 0.6 \\
\hline End of trial & $72.1 \pm 41.0$ & $63.1 \pm 28.8$ & $0.95^{\mathrm{a}}$ \\
\hline Difference between baseline and end-of-trial levels & $1.8(-27.5$ to 31.1$)$ & $-1.8(-13.4$ to 9.8$)$ & $0.67^{\mathrm{a}}$ \\
\hline \multicolumn{4}{|l|}{ Citrate (mg/24 h) } \\
\hline Baseline & $778.6 \pm 343.4$ & $606.9 \pm 248.6$ & 0.14 \\
\hline End of trial & $773.3 \pm 355.8$ & $603.9 \pm 268.1$ & 0.17 \\
\hline Difference between baseline and end-of-trial levels & $-5.3(-212.7$ to 202.0$)$ & $-3.0(-154.4$ to 148.4$)$ & 0.99 \\
\hline \multicolumn{4}{|l|}{ Oxalate (mg/24 h) } \\
\hline Baseline & $51.6 \pm 9.1$ & $54.7 \pm 11.2$ & $0.38^{\mathrm{a}}$ \\
\hline End of trial & $45.1 \pm 18.6$ & $42.5 \pm 17.8$ & 0.71 \\
\hline Difference between baseline and end-of-trial levels & $-6.5(-18.4$ to 5.5$)$ & $-12.2(-23.8$ to -0.7$)$ & 0.46 \\
\hline \multicolumn{4}{|l|}{ Uric acid (mg/24 h) } \\
\hline Baseline & $476.2 \pm 198.6$ & $536.3 \pm 195.9$ & 0.43 \\
\hline End of trial & $590.3 \pm 192.2$ & $598.9 \pm 220.9$ & 0.91 \\
\hline Difference between baseline and end-of-trial levels & $114.1(-27.3$ to 255.4$)$ & $62.6(-75.4$ to 200.6$)$ & 0.58 \\
\hline \multicolumn{4}{|l|}{$\mathrm{CaOxSS}$} \\
\hline Baseline & $7.39 \pm 2.90$ & $8.55 \pm 2.08$ & 0.23 \\
\hline End of trial & $7.06 \pm 4.01$ & $5.49 \pm 3.29$ & $0.33^{\mathrm{a}}$ \\
\hline Difference between baseline and end-of-trial levels & $-0.32(-3.17$ to 2.52$)$ & $-3.06(-5.45$ to -0.67$)$ & 0.12 \\
\hline \multicolumn{4}{|l|}{ ( } \\
\hline Baseline & $0.48 \pm 0.88$ & $0.42 \pm 0.60$ & $0.84^{\mathrm{a}}$ \\
\hline End of trial & $0.33 \pm 0.63$ & $0.78 \pm 1.43$ & $0.98^{\mathrm{a}}$ \\
\hline Difference between baseline and end-of-trial levels & $-0.15(-0.33$ to 0.04$)$ & $0.36(-0.57$ to 1.28$)$ & $0.54^{\mathrm{a}}$ \\
\hline \multicolumn{4}{|l|}{ UASS } \\
\hline Baseline & $1.08 \pm 0.71$ & $1.36 \pm 0.82$ & 0.35 \\
\hline End of trial & $1.66 \pm 1.00$ & $1.28 \pm 0.76$ & $0.40^{\mathrm{a}}$ \\
\hline Difference between baseline and end-of-trial levels & $0.58(-0.07$ to 1.24$)$ & $-0.08(-0.76$ to 0.60$)$ & 0.14 \\
\hline
\end{tabular}

Values are presented as mean \pm standard deviation or $95 \%$ confidence interval.

$\mathrm{CaOxSS}$, calcium oxalate supersaturation; CaPSS, calcium phosphate supersaturation; UASS, uric acid supersaturation.

${ }^{\mathrm{a}}$ :Nonparametric analysis. 
Table 4. Results of urine malondialdehyde to creatinine ratio and urine neutrophil gelatinase-associated lipocalin to creatinine ratio in the first morning urine sample before and after intervention

\begin{tabular}{|c|c|c|c|}
\hline Variable & Placebo group $(n=14)$ & Atorvastatin group $(n=14)$ & p-value \\
\hline \multicolumn{4}{|l|}{ U-MDA/Cr (nmol/g) } \\
\hline Baseline & $4.14 \pm 0.71$ & $4.13 \pm 0.90$ & 0.99 \\
\hline End of trial & $4.53 \pm 1.11$ & $4.37 \pm 1.33$ & $0.57^{\mathrm{a}}$ \\
\hline Difference between baseline and end-of-trial levels & $0.39(-0.32$ to 1.11$)$ & $0.24(-0.61$ to 1.09$)$ & 0.77 \\
\hline \multicolumn{4}{|l|}{ U-NGAL/Cr (ng/g) } \\
\hline Baseline & $42.27 \pm 80.85$ & $33.29 \pm 49.53$ & $0.15^{\mathrm{a}}$ \\
\hline End of trial & $32.10 \pm 57.85$ & $60.15 \pm 86.09$ & $0.98^{\mathrm{a}}$ \\
\hline Difference between baseline and end-of-trial levels & $-10.17(-27.56$ to 7.22$)$ & $26.86(-10.07$ to 63.79$)$ & $0.11^{\mathrm{a}}$ \\
\hline
\end{tabular}

U-MDA, urinary malondialdehyde; $\mathrm{Cr}$, creatinine; U-NGAL, urinary neutrophil gelatinase-associated lipocalin.

${ }^{\mathrm{a}}$ :Nonparametric analysis.

ancy between experimental animal and human studies can be attributed to differences in the sensitivity of the abovementioned biomarkers for the detection of study outcomes. In our study, we measured MDA based on a thiobarbituric acid reactive substances assay, which is a relatively easy and cost-effective method that is widely used across studies. However, the accuracy and sensitivity of this method for the detection of minute changes remains controversial [13]. Another biomarker evaluated in our study is NGAL, which is a tubular injury marker used in studies investigating kidney stones [23,24] or acute renal failure [27]. Previous research has shown that the level of urinary NGAL is higher in patients with urolithiasis than in healthy individuals [23,24], However, a recent study reported that NGAL may not be useful for the follow-up of renal tubular injury in cases of childhood urolithiasis [28]. Further research is warranted to define the specificity and sensitivity of these biomarkers to accurately follow-up changes in oxidative stress and renal tubular injury in adults with kidney stones.

Following are the limitations of our study in addition to those mentioned earlier: (1) The small sample size reduces the statistical power of our study and limits the ability to accurately detect the effect of our intervention. (2) Lack of serum parameters to measure oxidative stress and the use of a single biomarker to evaluate oxidative stress or tubular injury serve as other drawbacks of this research. Further clinical trials with a longer follow-up period, different drug doses, age- and sex stratification of patients and measurement of multiple biomarkers of oxidative stress and tubular injury are warranted to investigate the preventive effect of atorvastatin on kidney stone formation.

\section{CONCLUSIONS}

Atorvastatin administration at a dose of $20 \mathrm{mg} / \mathrm{d}$ for
3 months did not affect 24-h urinary metabolites, urinary MDA and NGAL in recurrent calcium stone formers. However, this study could not disprove the preventive role of atorvastatin in kidney stone formation. Future studies with a larger sample size, longer follow-up period, different drug doses and measurement of multiple biomarkers of oxidative stress and tubular injury are warranted to gain a deeper understanding of this important issue.

\section{CONFLICTS OF INTEREST}

The authors have nothing to disclose.

\section{ACKNOWLEDGMENTS}

This work was supported by the Urology and Nephrology Research Center, Shahid Beheshti University of Medical Sciences, Tehran, Iran, under Grant number UNRC.93.617.

\section{REFERENCES}

1. Pourmand G, Pourmand B. Epidemiology of stone disease in Iran. In: Talati JJ, Tiselius HG, Albala DM, Ye Z. Urolithiasis: basic science and clinical practice. London: Springer; 2012;857.

2. Masterson JH, Woo JR, Chang DC, Chi T, L'Esperance JO, Stoller ML, et al. Dyslipidemia is associated with an increased risk of nephrolithiasis. Urolithiasis 2015;43:49-53.

3. Sur RL, Masterson JH, Palazzi KL, L'Esperance JO, Auge BK, Chang DC, et al. Impact of statins on nephrolithiasis in hyperlipidemic patients: a 10-year review of an equal access health care system. Clin Nephrol 2013;79:351-5.

4. Cohen AJ, Adamsky MA, Nottingham CU, Pruitt J, Lapin B, Wang $\mathrm{CH}$, et al. Impact of statin intake on kidney stone formation. Urology 2019;124:57-61. 
5. Tsujihata M, Momohara C, Yoshioka I, Tsujimura A, Nonomura N, Okuyama A. Atorvastatin inhibits renal crystal retention in a rat stone forming model. J Urol 2008;180:2212-7.

6. Tsujihata M, Yoshioka I, Tsujimura A, Nonomura N, Okuyama A. Why does atorvastatin inhibit renal crystal retention? Urol Res 2011;39:379-83.

7. Temiz MZ, Yuruk E, Ertas K, Zengi O, Semercioz A. Effects of statin treatment with atorvastatin on urolithiasis-associated urinary metabolic risk factors: an experimental study. Int Urol Nephrol 2018;50:231-6.

8. Kumar A, Hecht C, Priyamvada S, Anbazhagan AN, Alakkam A, Borthakur A, et al. Probiotic Bifidobacterium species stimulate human SLC26A3 gene function and expression in intestinal epithelial cells. Am J Physiol Cell Physiol 2014;307:C108492.

9. Chow SC, Shao J, Wang H. Sample size calculations in clinical research. 2nd ed. Boca Raton: Taylor \& Francis; 2007;60.

10. Skolarikos A, Straub M, Knoll T, Sarica K, Seitz C, Petřík A, et al. Metabolic evaluation and recurrence prevention for urinary stone patients: EAU guidelines. Eur Urol 2015;67:750-63.

11. Farmer KC. Methods for measuring and monitoring medication regimen adherence in clinical trials and clinical practice. Clin Ther 1999;21:1074-90.

12. Noori N, Honarkar E, Goldfarb DS, Kalantar-Zadeh K, Taheri $\mathrm{M}$, Shakhssalim N, et al. Urinary lithogenic risk profile in recurrent stone formers with hyperoxaluria: a randomized controlled trial comparing DASH (Dietary Approaches to Stop Hypertension)-style and low-oxalate diets. Am J Kidney Dis 2014;63:456-63.

13. Satoh K. Serum lipid peroxide in cerebrovascular disorders determined by a new colorimetric method. Clin Chim Acta 1978;90:37-43.

14. Tavasoli S, Basiri A, Khoshdel A, Taheri M. Evaluating the association of body mass index, waist circumference and waist to stature ratio with urine composition in patients with urolithiasis. Iran J Kidney Dis 2017;11:371-8.

15. Santhosh Kumar M, Selvam R. Supplementation of vitamin E and selenium prevents hyperoxaluria in experimental urolithic rats. J Nutr Biochem 2003;14:306-13.

16. Fry DW, Richardson KE. Isolation and characterization of glycolic acid oxidase from human liver. Biochim Biophys Acta 1979;568:135-44.

17. Steinhubl SR. Why have antioxidants failed in clinical trials?
Am J Cardiol 2008;101(10A):14D-9D.

18. Parthasarathy S, Khan-Merchant N, Penumetcha M, Khan BV, Santanam N. Did the antioxidant trials fail to validate the oxidation hypothesis? Curr Atheroscler Rep 2001;3:392-8.

19. Kok DJ, Boellaard W, Ridwan Y, Levchenko VA. Timelines of the "free-particle" and "fixed-particle" models of stone-formation: theoretical and experimental investigations. Urolithiasis 2017;45:33-41.

20. Ma MC, Chen YS, Huang HS. Erythrocyte oxidative stress in patients with calcium oxalate stones correlates with stone size and renal tubular damage. Urology 2014;83:510.e9-17.

21. Boonla C, Wunsuwan R, Tungsanga K, Tosukhowong P. Urinary 8-hydroxydeoxyguanosine is elevated in patients with nephrolithiasis. Urol Res 2007;35:185-91.

22. Carnevale R, Pignatelli P, Di Santo S, Bartimoccia S, Sanguigni $\mathrm{V}$, Napoleone L, et al. Atorvastatin inhibits oxidative stress via adiponectin-mediated NADPH oxidase down-regulation in hypercholesterolemic patients. Atherosclerosis 2010;213:22534.

23. Zhu W, Liu M, Wang GC, Che JP, Xu YF, Peng B, et al. Urinary neutrophil gelatinase-associated lipocalin, a biomarker for systemic inflammatory response syndrome in patients with nephrolithiasis. J Surg Res 2014;187:237-43.

24. Kandur Y, Gonen S, Fidan K, Soylemezoglu O. Evaluation of urinary KIM-1, NGAL, and IL-18 levels in determining early renal injury in pediatric cases with hypercalciuria and/or renal calculi. Clin Nephrol 2016;86:62-9.

25. Carrasco-Valiente J, Anglada-Curado FJ, Aguilar-Melero P, González-Ojeda R, Muntané-Relat J, Padillo-Ruiz FJ, et al. [State of acute phase markers and oxidative stress in patients with kidney stones in the urinary tract]. Actas Urol Esp 2012;36:296-301. Spanish.

26. Tungsanga K, Sriboonlue P, Futrakul P, Yachantha C, Tosukhowong P. Renal tubular cell damage and oxidative stress in renal stone patients and the effect of potassium citrate treatment. Urol Res 2005;33:65-9.

27. Devarajan P. Neutrophil gelatinase-associated lipocalin: a promising biomarker for human acute kidney injury. Biomark Med 2010;4:265-80.

28. Taşdemir M, Fuçucuoğlu D, Küçük SH, Erol M, Yiğit Ö, Bilge I. Urinary biomarkers in the early detection and follow-up of tubular injury in childhood urolithiasis. Clin Exp Nephrol 2018;22:133-41. 